\title{
AN INTRODUCTION TO REDUCED BASIS METHOD FOR PARAMETRIZED PDEs
}

\author{
GIANLUIGI ROZZA* \\ Ecole Polytechnique Fédérale de Lausanne \\ Chair of Modelling and Scientific Computing \\ Station 8-MA, CH-1015 Lausanne, Switzerland \\ E-mail: gianluigi.rozza@epfl.ch \\ http://iacs.epfl.ch/ rozza
}

\begin{abstract}
We provide an introduction on reduced basis (RB) method for the solution of parametrized partial differential equations (PDEs). We introduce all the main ingredients to describe the methodology and the algorithms used to build the approximation spaces and the error bounds. We consider a model problem describing a steady potential flow around parametrized bodies and we provide some illustrative results.
\end{abstract}

Keywords: Reduced basis method; parametrized PDEs; Galerkin method; error bounds; potential flows.

\section{Introduction}

In several optimization contexts arising, for example, in the aerospace industry, the problem of the resolution of PDEs in parametrized configurations is growing. The necessity to avoid to rebuild the geometry for each simulation and to have real-time computations in the many-query context provides a strong motivation for the development of the reduced basis method (Refs. 1-6) as a tool for the solution of parametrized problem built upon finite element (FE) method (Ref. 7).

In Sec. 2 we define a model problem, then in Sec. 3 we provide some basic results for the introduction of the methodology. Sec. 4 and Sec. 5 are devoted for the introduction of lower bounds for the coercivity constant and for a posteriori error bounds, respectively. In Sec. 6 we present numerical results considering steady potential flows around parametrized bodies, then

*Numerical simulations in collaboration with Gwenol Grandperrin (EPFL, Mathematics Section) 
some conclusions follow.

\section{Problem definition}

We introduce an abstract model problem. We consider $\mathcal{D} \subset \mathbb{R}^{p}$ as the range of variation of $p$ parameters and $\Omega \subset \mathbb{R}^{d}$ as a domain ( $p$ and $d$ are integers). The functional space $X^{e}$ is such that $\left(H_{0}^{1}(\Omega)\right) \subset X^{e} \subset\left(H^{1}(\Omega)\right)$, with $H^{1}(\Omega)$ the Sobolev space defined as $H^{1}(\Omega)=\left\{f \in L^{2}(\Omega) \mid D^{\alpha} f \in\right.$ $\left.L^{2}(\Omega), \alpha \leq 1\right\}$ and $D^{\alpha} f$ derivation of $f$ in the distributions sense, $L^{2}(\Omega)=$ $\left\{f: \Omega \rightarrow \mid \int_{\Omega} f(x)^{2} d x<\infty\right\} ; H_{0}^{1}(\Omega)=\left\{f \in H^{1}(\Omega) \mid f=0\right.$ on $\left.\partial \Omega\right\}$ and $H_{D}^{1}=\left\{f \in H^{1} \mid f=0\right.$ on $\left.\Gamma_{D}, D \subset \partial \Omega\right\}$.

We introduce $\forall \mu \in \mathcal{D}$ a bilinear and coercive form $a(., . ; \mu)$ and two linear and continuos functionals $f(. ; \mu)$ and $l(. ; \mu)$, then we consider the following "exact" problem

$$
\left\{\begin{array}{l}
\text { For } \mu \in \mathcal{D}, \text { solve } \\
s^{e}(\mu)=l\left(u^{e}(\mu) ; \mu\right) \text { s.t. } u^{e}(\mu) \in X^{e} \text { satisfies } \\
a\left(u^{e}, v ; \mu\right)=f(v ; \mu) \quad \forall v \in X^{e} .
\end{array}\right.
$$

We define a scalar product and a norm related with the energy of the system as: $\langle w, v\rangle_{\mu} \equiv a(w, v ; \mu)$, and $\|\mid w\|_{\mu} \equiv\langle w, w\rangle_{\mu}^{\frac{1}{2}} \forall w, v \in X^{e}$, respectively. We also introduce a second scalar product and its norm defined on $X^{e}(\tau>0)$ for a selected $\bar{\mu}$ such that (Ref. 6) $(w, v)_{X} \equiv\langle w, v\rangle_{\bar{\mu}}+\tau(w, v)_{L^{2}(\Omega)}$, and $\|w\|_{X} \equiv(w, w)_{X}^{\frac{1}{2}} \quad \forall w, v \in X^{e}$, respectively. We introduce the crucial hypothesis that the bilinear form $a$ can be expressed as

$$
a(w, v ; \mu)=\sum_{q=1}^{Q} \Theta^{q}(\mu) a^{q}(w, v)
$$

such that for $q=1, \ldots, Q: \Theta^{q}: \mathcal{C} \rightarrow \mathbb{R}$ is depending on $\mu$ and $a^{q}: X^{e} \times X^{e} \rightarrow \mathbb{R}$ is $\mu$-independent. ${ }^{2}$ This hypothesis on $a$ allows us to significantly improve the computational efficiency in the evaluation of $a(w, v ; \mu)$ : the component $a_{q}(w, v)$ can be computed once and then stored to form (2). We are interested in geometrical parametrizations such that $\Omega$ will be a reference (and fixed) domain and it can be seen as the preimage of $\Omega_{0}(\mu)$ (depending on the parameters) denoted original domain through the transformation $\mathcal{T}: \Omega \rightarrow \Omega_{0}$. We can define $a_{0}\left(w_{0}, v_{0} ; \mu\right)$ as $a(w, v ; \mu)=a_{0}(\mathcal{T}(w), \mathcal{T}(v) ; \mu)$.

We introduce a numerical discretization in our model problem given by finite element method such that the space $X^{\mathcal{N}} \subset X^{e}\left(\operatorname{dim}\left(X^{\mathcal{N}}\right)=\mathcal{N}\right)$ and 
the problem is reformulated as

$$
\left\{\begin{aligned}
\text { For } \mu \in \mathcal{D}, & \text { solve } \\
s^{\mathcal{N}}(\mu) & =l\left(u^{\mathcal{N}}(\mu)\right) \text { and } u^{\mathcal{N}}(\mu) \in X^{\mathcal{N}} \text { satisfies } \\
a\left(u^{\mathcal{N}}, v ; \mu\right) & =f(v ; \mu) \quad \forall v \in X^{\mathcal{N}}
\end{aligned}\right.
$$

We recall the definition of the coercivity constant for the discretized problem as $\alpha^{\mathcal{N}}(\mu) \equiv \inf _{w \in X^{\mathcal{N}}} \frac{a(w, w ; \mu)}{\|w\|_{X}^{2}}, \forall \mu \in \mathcal{D}$.

\section{Reduced basis method}

We introduce a principal set of parameters $\Xi=\left\{\mu^{1}, \ldots, \mu^{N_{\max }}\right\} \subset \mathcal{D}$ and then for $1 \leq N \leq N_{\max }$ we define the subsets $S_{N}=\left\{\mu^{1}, \ldots, \mu^{N}\right\}$ to which we associate the Lagrange reduced basis space (see Refs. 2,5,6) defined as $W_{N}^{\mathcal{N}}=\operatorname{vect}\left\{u^{\mathcal{N}}\left(\mu^{n}\right), 1 \leq n \leq N\right\}$. It is clear that the nested (or hierarchical) condition is valid for $S_{1} \subset S_{2} \subset \ldots \subset S_{N_{\max }}$ and for $W_{1}^{\mathcal{N}} \subset W_{2}^{\mathcal{N}} \subset \ldots \subset W_{N_{\max }}^{\mathcal{N}} \subset X^{\mathcal{N}}$. The finite element solutions $u^{\mathcal{N}}\left(\mu^{n}\right)$ for $1 \leq n \leq N_{\max }$ and for some properly selected values of the parameter $\mu^{n}$ are referred to as snapshots. By a Galerkin projection we can solve the reduced basis problem defined as

$$
\left\{\begin{array}{c}
\text { For a new } \mu \in \mathcal{D} \text {, evaluate } \\
s_{N}^{\mathcal{N}}(\mu)=l\left(u_{N}^{\mathcal{N}}(\mu)\right) \text { s.t. } u_{N}^{\mathcal{N}}(\mu) \in W_{N}^{\mathcal{N}} \subset X^{\mathcal{N}} \text { satisfies } \\
a\left(u_{N}^{\mathcal{N}}, v ; \mu\right)=f(v) \quad \forall v \in W_{N}^{\mathcal{N}} .
\end{array}\right.
$$

The goal is to obtain a cheap evaluation of $s_{N}^{\mathcal{N}}(\mu)$ for many values of $\mu$. Lemma 3.1 shows that $s_{N}^{\mathcal{N}}$ is converging to $s^{\mathcal{N}}$ quadratically with respect to the convergence ${ }^{6}$ of $u_{N}^{\mathcal{N}}$ to $u^{\mathcal{N}}$.

Lemma 3.1. Best approximation of $R B$ method and quadratic convergence of the output for $l=f$ (compliance, see Ref. 2) is given by:

(1) $\left\|\left|u^{\mathcal{N}}(\mu)-u_{N}^{\mathcal{N}}(\mu)\|\|_{\mu} \leq \inf _{w \in X_{N}^{\mathcal{N}}}\left\|\mid u^{\mathcal{N}}(\mu)-w\right\|_{\mu}\right.\right.$;

(2) $s^{\mathcal{N}}(\mu)-s_{N}^{\mathcal{N}}(\mu)=\|\| u^{\mathcal{N}}(\mu)-u_{N}^{\mathcal{N}}(\mu)\|\|_{\mu}^{2}$.

When $l \neq f$ the "square" effect in the convergence is given by the solution of a dual problem. ${ }^{6}$ To have a system from (4) which is not ill-conditioned we can use a Gram-Schmidt orthonomalization procedure (see Ref. 2) for the snapshots $u^{\mathcal{N}}\left(\mu^{n}\right), 1 \leq n \leq N_{\max }$, with respect to the scalar product $(\cdot, \cdot)_{X}$ to obtain $\zeta_{n}^{\mathcal{N}}, 1 \leq n \leq N_{\max }$ as basis functions, so that $W_{N}^{\mathcal{N}}=\left\{\zeta_{n}^{\mathcal{N}}\right\}_{n=1, \ldots, N}$ for $1 \leq N \leq N_{\max }$. 
We can rewrite $u_{N}^{\mathcal{N}}$ as

$$
u_{N}^{\mathcal{N}}(\mu)=\sum_{m=1}^{N} u_{N m}^{\mathcal{N}}(\mu) \zeta_{m}^{\mathcal{N}}
$$

By posing $v=\zeta_{n}^{\mathcal{N}}, 1 \leq n \leq N$ in (4) we get

$$
\sum_{m=1}^{N} a\left(\zeta_{m}^{\mathcal{N}}, \zeta_{n}^{\mathcal{N}} ; \mu\right) u_{N m}^{\mathcal{N}}(\mu)=f\left(\zeta_{n}^{\mathcal{N}}\right) \quad 1 \leq n \leq N,
$$

and by (2) we may rewrite (6) as

$$
\sum_{m=1}^{N}\left(\sum_{q=1}^{Q} \Theta^{q}(\mu) a^{q}\left(\zeta_{m}^{\mathcal{N}}, \zeta_{n}^{\mathcal{N}}\right)\right) u_{N m}^{\mathcal{N}}(\mu)=f\left(\zeta_{n}^{\mathcal{N}}\right) \quad 1 \leq n \leq N .
$$

Here $\zeta^{\mathcal{N}}$ are independent by $\mu$ and so the quantities $f\left(\zeta_{n}^{\mathcal{N}}\right)\left(l\left(\zeta_{n}^{\mathcal{N}}\right)\right), 1 \leq n \leq$ $N_{\text {max }}$ and $a^{q}\left(\zeta_{m}^{\mathcal{N}}, \zeta_{n}^{\mathcal{N}}\right), 1 \leq n \leq N_{\text {max }}, 1 \leq q \leq Q$ can be pre-computed and stored to decouple the offline computational part (parameter independent) from the online one (parameter dependent). The output can be computed as $s_{N}^{\mathcal{N}}=\sum_{m=1}^{N} u_{N m}^{\mathcal{N}}(\mu) l\left(\zeta_{m}^{\mathcal{N}}\right)$.

\subsection{Greedy algorithm for reduced basis space construction}

Let $\Xi$ a subset of $\mathcal{D}$, used as a surrogate of $\mathcal{D}$ to test the reduced basis approximation. This subset has to be sufficiently rich and it can be built by using Monte-Carlo sampling (with uniform or log density, see Ref. 2).

In order to build the space $W_{N}^{\mathcal{N}}$, given $\Xi$ and $N_{\max }$ we start by considering $S_{1}=\left\{\mu^{1}\right\}, W_{1}^{\mathcal{N}}=\operatorname{span}\left\{u^{\mathcal{N}}\left(\mu^{1}\right)\right\}$. Then, for $N=2, \ldots, N_{\text {max }}$, we look for

$$
\mu^{N}=\arg \max _{\mu \in \Xi} \Delta_{N-1}(\mu)
$$

where $\Delta_{N}=\Delta_{N}^{e n}$ or $\Delta_{N}^{s}$ are the error bounds for the energy or the output $s_{N}^{\mathcal{N}}$, respectively. These quantities will be introduced in Sec. 5. Instead of fixing $N_{\max }$ it is possible to set a tolerance $\epsilon$ as stopping criterium for a new $\mu^{N}$ when $\max _{\mu \in \Xi} \Delta_{N-1}(\mu) \leq \epsilon$. We define, in a recursive way, $S_{N}=$ $S_{N-1} \cup \mu^{N}$ and $W_{N}^{\mathcal{N}}=W_{N-1}^{\mathcal{N}} \cup \operatorname{span}\left\{u^{\mathcal{N}}\left(\mu^{N}\right)\right\}$. In this way only few FE solutions have to be computed (just the selected snapshots).

\section{Lower bounds for the coercivity constant}

We are interested in getting a fast and reliable method to compute a lower bound for the corcivity constant which is going to play a role in the error 
bounds. We use the so-called successive constraint method (SCM), briefly recalled here, see Refs. 6,8. This method uses online a linear programming algorithm with a number of operations independent by $\mathcal{N}$.

Let us introduce an objective function $\mathcal{J}^{\text {obj }}: \mathcal{D} \times \mathbb{R}^{Q} \rightarrow \mathbb{R}$, s.t. $(\mu, y) \mapsto \sum_{q=1}^{Q} \Theta^{q}(\mu) y_{q}$ where $y=\left(y_{1}, \ldots, y_{Q}\right)$. The coercivity constant can be expressed as $\alpha^{\mathcal{N}}(\mu)=\inf _{y \in \mathcal{Y}} \mathcal{J}^{\text {obj }}(\mu ; y)$ where $\mathcal{Y}=$ $\left\{y \in \mathbb{R}^{Q} \mid \exists w_{y} \in X^{\mathcal{N}}\right.$ such that $\left.y_{q}=\frac{a^{q}\left(w_{y}, w_{y}\right)}{\left\|w_{y}\right\|_{X}^{2}}, 1 \leq q \leq Q\right\}$. We introduce also a box of constraints

$$
\mathcal{B}=\prod_{q=1}^{Q}\left[\inf _{w \in X^{\mathcal{N}}} \frac{a^{q}(w, w)}{\|w\|_{X}^{2}}, \sup _{w \in X^{\mathcal{N}}} \frac{a^{q}(w, w)}{\|w\|_{X}^{2}}\right] .
$$

We properly select a set of parameters in $\mathcal{D}$ denoted with $C_{J}=$ $\left\{\mu_{S C M}^{1} \in \mathcal{D}, \ldots, \mu_{S C M}^{J} \in \mathcal{D}\right\}$, and we indicate with $C_{J}^{M, \mu}$ the set of $M \geq 1$ elements of $C_{J}$ nearest $\mu \in \mathcal{D}$; if $M>J$ then $C_{J}^{M, \mu}=C_{J}$. There are some techniques to build $C_{J}$ as reported in Ref. 8. Given $C_{J}, M \in \mathbb{N}=\{1,2, \ldots\}$ and $\mu \in \mathcal{D}$, we define $\mathcal{Y}_{L B}\left(\mu ; C_{J}, M\right)$ as

$$
\mathcal{Y}_{L B}\left(\mu ; C_{J}, M\right)=\left\{y \in \mathbb{R}^{Q} \mid y \in \mathcal{B}, \mathcal{J}^{o b j}\left(\mu^{\prime}, y\right) \geq \alpha^{\mathcal{N}}\left(\mu^{\prime}\right), \forall \mu^{\prime} \in C_{J}^{M, \mu}\right\},
$$

such that $\mathcal{Y} \subset \mathcal{Y}_{L B}\left(\mu ; C_{J}, M\right) \quad \forall \mu \in \mathcal{D}$ (being $\mathcal{Y}_{L B}$ richer than $\mathcal{Y}$ ) and we define the lower bound of the coercity constant as $\alpha_{L B}^{\mathcal{N}}\left(\mu ; C_{J}, M\right)=$ $\min _{y \in \mathcal{Y}_{L B}\left(\mu ; C_{J}, M\right)} \mathcal{J}^{o b j}(\mu, y)$ such that $\alpha_{L B}^{\mathcal{N}}(\mu) \leq \alpha^{\mathcal{N}}(\mu), \forall \mu \in \mathcal{D}$. The problem of computing a lower bound for the coercivity constant $\alpha_{L B}^{\mathcal{N}}$ is a linear programming minimization problem with $Q$ variables $y_{1}, \ldots, y_{Q}$ and $2 Q+M$ constraints. Each $y_{i}$ is subject to two constraints from $\mathcal{B}(8)$ and then there are the $M$ conditions $\mathcal{J}^{\text {obj }}\left(\mu^{\prime}, y\right) \geq \alpha^{\mathcal{N}}\left(\mu^{\prime}\right), \forall \mu^{\prime} \in C_{J}^{M, \mu}$ and $\left|C_{j}^{M, \mu}\right| \leq M$. Given $\mathcal{B}$ and $\left\{\alpha^{\mathcal{N}}\left(\mu^{\prime}\right) \mid \mu^{\prime} \in C_{J}\right\}$, the evaluation of $\mu \rightarrow \alpha_{L B}^{\mathcal{N}}(\mu)$ is independent by $\mathcal{N}$.

\section{A posteriori error estimation}

We introduce here the a posteriori error bounds as described in Refs. 2,6,9. We are interested in a method which should be realible and efficient. We reconsider the finite element problem (3) and the Galerkin projection to get the problem (4). We define $e(\mu) \equiv u^{\mathcal{N}}(\mu)-u_{N}^{\mathcal{N}}(\mu) \in X^{\mathcal{N}}$. Thanks to the linearity of $a(.,$.$) we have$

$$
\begin{aligned}
a(e(\mu), v ; \mu) & =a\left(u^{\mathcal{N}}(\mu), v ; \mu\right)-a\left(u_{N}^{\mathcal{N}}(\mu), v ; \mu\right) \\
& =f(v ; \mu)-a\left(u_{N}^{\mathcal{N}}(\mu), v ; \mu\right) \quad \forall v \in X^{\mathcal{N}} .
\end{aligned}
$$


We denote

$$
r(v ; \mu)=f(v ; \mu)-a\left(u_{N}^{\mathcal{N}}(\mu), v ; \mu\right),
$$

to get the equation of the residual

$$
a(e(\mu), v ; \mu)=r(v ; \mu) .
$$

Thanks to Riesz representation theorem we can write $r(v ; \mu)$ as

$$
r(v ; \mu)=(\hat{e}(\mu), v)_{X} \quad \forall v \in X^{\mathcal{N}},
$$

so that from (10) we get $a(e(\mu), v ; \mu)=(\hat{e}(\mu), v)_{X}$. At the same time we have $\|r(. ; \mu)\|_{\left(X^{\mathcal{N}}\right)^{\prime}} \equiv \frac{r(v ; \mu)}{\|v\|_{X}}=\|\hat{e}(\mu)\|_{X}$. By the coercivity lower bound $\alpha_{L B}^{\mathcal{N}}(\mu)$ introduced in Sec. 4 we define the following error bound for the energy norm

$$
\Delta_{N}^{e n}(\mu)=\frac{\|\hat{e}(\mu)\|_{X}}{\sqrt{\alpha_{L B}^{\mathcal{N}}(\mu)}}
$$

and for the output

$$
\Delta_{N}^{s}(\mu)=\frac{\|\hat{e}(\mu)\|_{X}^{2}}{\alpha_{L B}^{\mathcal{N}}(\mu)} .
$$

Concerning $\Delta_{N}^{s}$, if $l=f$ we have also that $\left|s^{\mathcal{N}}(\mu)-s_{N}^{\mathcal{N}}(\mu)\right| \leq \Delta_{N}^{s}=\left(\Delta_{N}^{e n}\right)^{2}$ for $N=1, \ldots, N_{\max }$ and for all $\mu \in \mathcal{D}$. See Ref. 6 for demonstrations.

\subsection{Offline-online computational procedures}

The residual (9) can be computed as

$$
\begin{aligned}
r(v ; \mu) & \equiv f(v)-a\left(u_{N}^{\mathcal{N}}(\mu), v ; \mu\right) \\
& =f(v)-\sum_{n=1}^{N} u_{N}^{\mathcal{N}}(\mu) \sum_{q=1}^{Q} \Theta^{q}(\mu) a^{q}\left(\zeta_{n}^{\mathcal{N}}, v\right) \text { by }(5)-(2)
\end{aligned}
$$

by (11) we get $(\hat{e}(\mu), v)_{X} \equiv r(v ; \mu)$

$$
=f(v)-\sum_{n=1}^{N} \sum_{q=1}^{Q} \Theta^{q}(\mu) u_{N}^{\mathcal{N}}(\mu) a^{q}\left(\zeta_{n}^{\mathcal{N}}, v\right) .
$$

We may rewrite $\hat{e}(\mu)$ as $\hat{e}(\mu)=\mathcal{C}+\sum_{q=1}^{Q} \sum_{n=1}^{N} \Theta^{q}(\mu) u_{N_{n}}^{\mathcal{N}}(\mu) a^{q}\left(\zeta_{n}^{\mathcal{N}}, v\right)$ where $\mathcal{C}$ and $\mathcal{L}_{n}^{q}, 1 \leq n \leq N, 1 \leq q \leq Q$ are given by the following problems

$$
\left\{\begin{aligned}
(\mathcal{C}, v)_{X} & =f(v) \quad \forall v \in X^{\mathcal{N}} \\
\left(\mathcal{L}_{n}^{q}, v\right)_{X} & =-a^{q}\left(\zeta_{n}^{\mathcal{N}}, v\right) \quad \forall v \in X^{\mathcal{N}}
\end{aligned}\right.
$$


The quantity $\|\hat{e}(\mu)\|_{X}^{2}$ is then given by

$$
\begin{array}{r}
\|\hat{e}(\mu)\|_{X}^{2}=(\mathcal{C}, \mathcal{C})_{X}+2 \sum_{q=1}^{Q} \sum_{n=1}^{N} \Theta^{q}(\mu) u_{N_{n}}^{\mathcal{N}}(\mu)\left(\mathcal{C}, \mathcal{L}_{n}^{q}\right)_{X} \\
+\sum_{q=1}^{Q} \sum_{n=1}^{N} \Theta^{q}(\mu) u_{N_{n}}^{\mathcal{N}}(\mu)\left\{\sum_{q^{\prime}=1}^{Q} \sum_{n^{\prime}=1}^{N} \Theta^{q}(\mu) u_{N_{n}}^{\mathcal{N}}(\mu)\left(\mathcal{L}_{n}^{q}, \mathcal{L}_{n^{\prime}}^{q^{\prime}}\right)_{X}\right\} .
\end{array}
$$

In the offline part, we compute quantities $\mu$-independent like $\mathcal{C}, \mathcal{L}_{n}^{q}, 1 \leq$ $n \leq N_{\max }, 1 \leq q \leq Q$ and we store $(\mathcal{C}, \mathcal{C})_{X},\left(\mathcal{C}, \mathcal{L}_{n}^{q}\right)_{X},\left(\mathcal{L}_{n}^{q}, \mathcal{L}_{n^{\prime}}^{q^{\prime}}\right)_{X} \quad 1 \leq$ $n, n^{\prime} \leq N_{\max }, 1 \leq q, q^{\prime} \leq Q$. In the offline part, the computational complexity depends on $N_{\max }, Q$ and $\mathcal{N}$. In the online part we compute the $\mu$-dependent quantities $\Theta^{q}(\mu), 1 \leq q \leq Q, u_{N n}^{\mathcal{N}}(\mu), 1 \leq n \leq N$. To evaluate (15) we perform $O\left(Q^{2} N^{2}\right)$ operations, independently by $\mathcal{N}$.

\section{Numerical results}

We present here some numerical results as example of application of reduced basis method to potential flows around parametrized bodies (see Ref. 10), representing for example the bulb of a yacht (with or without the keel) or the nacelles of an aircraft or bodies placed under the wings and/or the fuselage. We provide the general parametrization, state equation, convergence results and some representative solutions. Our emphasys is on computational performance.

\subsection{Bulb and keel}

In Fig. 1 we report two very preliminary configurations used as a parametrized bodies for our first tests. The first configuration, describing just the bulb without the keel, has three parameters $\mu_{1}, \mu_{2}, \mu_{3}$ for the nonsymmetric ellipse, the second configuration, with the inclusion of the keel in the bulb configuration has five parameters including a parametrization on the keel height and width.

In Fig. 2 we report the two domains used for this first tests. We used a simple inviscid and irrotational fluid model described by a potential flow (Ref. 10) and represented by a Laplace problem, whose strong formulation 

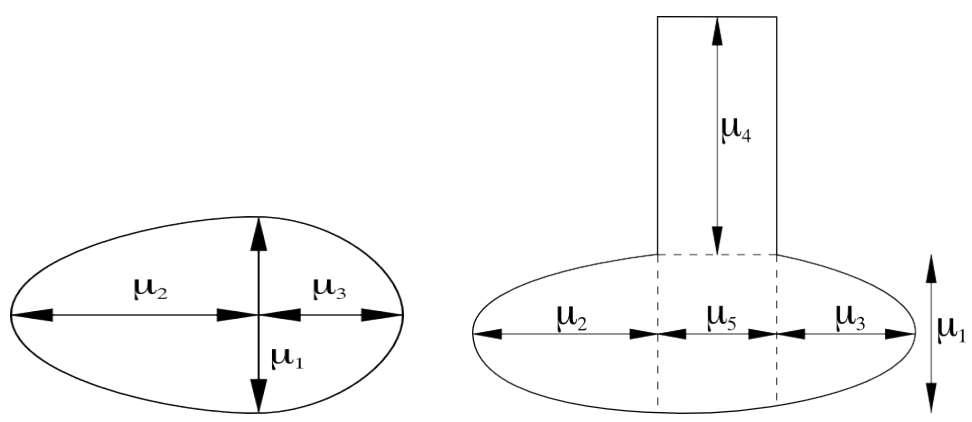

Fig. 1. Parametrized bodies: bulb (left) and keel with bulb (right).

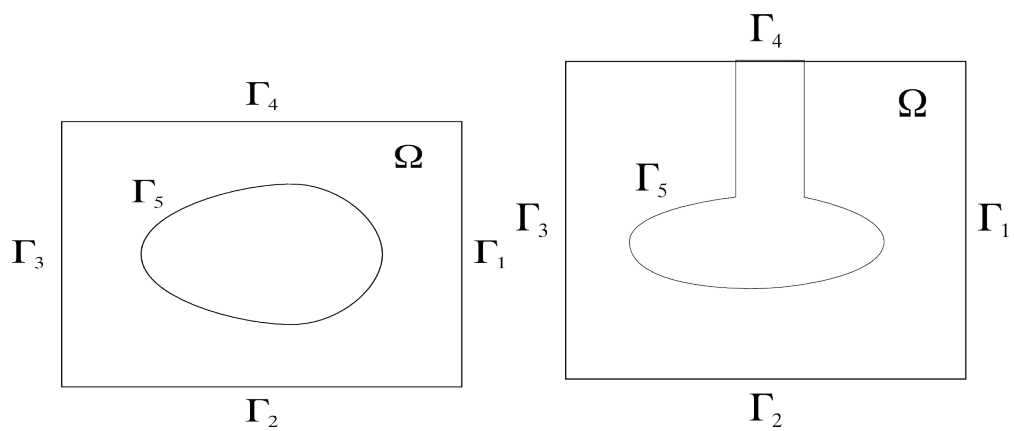

Fig. 2. Domains for the bulb (left) and for the keel with the bulb (right).

is

$$
\left\{\begin{aligned}
\text { Find } u^{\mathcal{N}}(\mu) \in X^{\mathcal{N}} \text { s.t. } & \\
\Delta u^{\mathcal{N}}=0 & \text { on } \Omega \\
u^{\mathcal{N}}=1 & \text { on } \Gamma_{1} \\
\partial_{n} u^{\mathcal{N}}=1 & \text { on } \Gamma_{3} \\
\partial_{n} u^{\mathcal{N}}=0 & \text { on } \Gamma_{2} \cup \Gamma_{4} \cup \Gamma_{5},
\end{aligned}\right.
$$

where $\partial_{n}$ indicates the normal derivative. On the outflow $\Gamma_{1}$ we impose a non-homogeneous Dirichlet condition representing the potential level, on $\Gamma_{3}$ we impose a non-homogeneous Neumann condition representing the imposition of a (unity) velocity in the normal direction, on $\Gamma_{2}, \Gamma_{4}, \Gamma_{5}$ we impose a homogeneous Neumann condition representing a condition of zero velocity in the normal direction of the body and/or walls (non-penetration). The previous problem is then transfomed in the form introduced in (1). We report in Fig. 3 some convergence results plotting the quantity $\max \left(\Delta_{N}^{e n}\right)$ (12) over a very large sample $\Xi \subset \mathcal{D}$ as a function of $N$ during the greedy 
algorithm of Sec. 3.1 for the two test configurations. For the coercivity lower bounds of Sec. 4 we used an approximation with $J=169$ and $J=187$, respectively for the bulb and for the bulb with the keel. The parameters range is given by $\mathcal{D}=[1,4] \times[1,4] \times[1,4]$ for the first example (bulb) and by $\mathcal{D}=[1,7] \times[1,7] \times[1,7] \times[1,8] \times[1,5]$ for the second one (keel and bulb). Computational results were performed using the library rbMIT. ${ }^{11}$
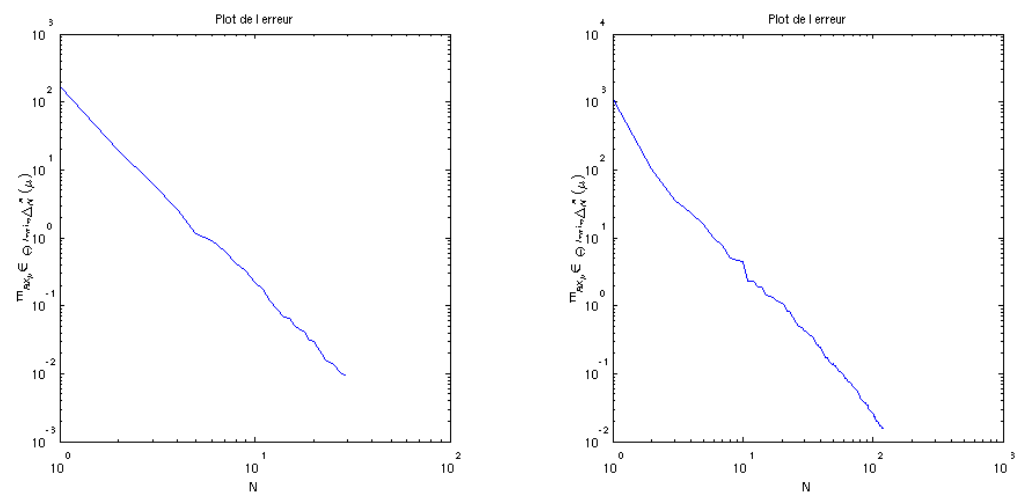

Fig. 3. Very fast convergence of the greedy algorithm over a large sample of parameters: maximum $\Delta_{N}^{e n}(12)$ as a function of $N$ for the bulb configuration (left) and for the keel and the bulb (right).

We report in Fig. 4 representative solutions of the bulb problem for a reference value of $\mu=[3,4,3]$. We report the potential solution with over the velocity field (on the left) and the pressure field (on the right) computed by the Bernoulli Theorem. ${ }^{10}$
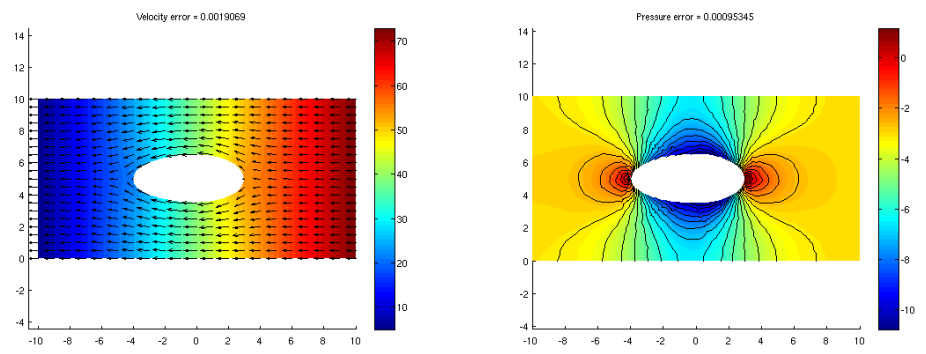

Fig. 4. A representative solution of velocity and potential (left) and pressure (right) for the bulb test. 
Thanks to reduced basis method we can get real-time evaluations and visualizations of parametrized problems by testing a large number of different configurations, corresponding to many $\mu$ 's belonging to $\mathcal{D}$. In Table 1 we report offline and online computational times for the two first tests. We can see that the average online computational times for the solution of the problem for a certain $\mu \in \mathcal{D}$ (with error bounds) is less than $1 \%$ compared with the offline computational times needed to set the geometry, build the mesh and compute FE solutions (and error bound ingredients preparation).

Table 1. Computational times for the two preliminary tests and comparison.

\begin{tabular}{|c|c|c|}
\hline test & bulb & bulb and keel \\
\hline phase & computational times $(\mathrm{s})$ & computational times $(\mathrm{s})$ \\
\hline offline & 553.8 & 763.4 \\
online & 5.2 & 7.4 \\
\hline ratio & $0.94 \%$ & $0.97 \%$ \\
\hline
\end{tabular}

\subsection{A more complex example: several parametrized bodies}

We consider here a more complex example where we study a parametrized configuration made up by three bodies with different shape, size and position. This test can be seen as a preliminary study for a trimaran configuration, a multihulled boat or for an aircraft with many nacelles.

In Fig. 5 we report a scheme with 8 parameters considered: parameters $\mu_{1}-\mu_{6}$ are describing the bodies' geometry, which are non-symmetric ellipses (upper and lower bodies have the same parametrization $\mu_{4}-\mu_{6}$ ). Parameters $\mu_{7}$ and $\mu_{8}$ are responsible of the position of the bodies in the domain and their mutual distance ( horizontal and vertical distance). The range of variation of the parameters is given by $\mathcal{D}=[3,8] \times[3,20] \times[3,20] \times$ $[3,8] \times[3,20] \times[3,6] \times[3,8] \times[-8,8]$. In the same figure we illustrate the domain $\Omega$.

The state problem is the same as the one described in Sec. 6.1 where we considered steady potential flow; in this case we have just two more boundaries to consider: $\Gamma_{6}$ and $\Gamma_{7}$ with homogeneous Neumann condition (zero velocity). We report in Fig. 6 the mesh and triangulation of the considered configuration (using rbMIT $^{11}$ ) and some convergence results plotting the quantity $\max \left(\Delta_{N}^{e n}\right)(12)$ over a very large sample $\Xi \subset \mathcal{D}$ as a function of $N$, during the greedy algorithm of Sec. 3.1. For the coercivity lower bounds of Sec. 4 we used an approximation with $J=200$. 


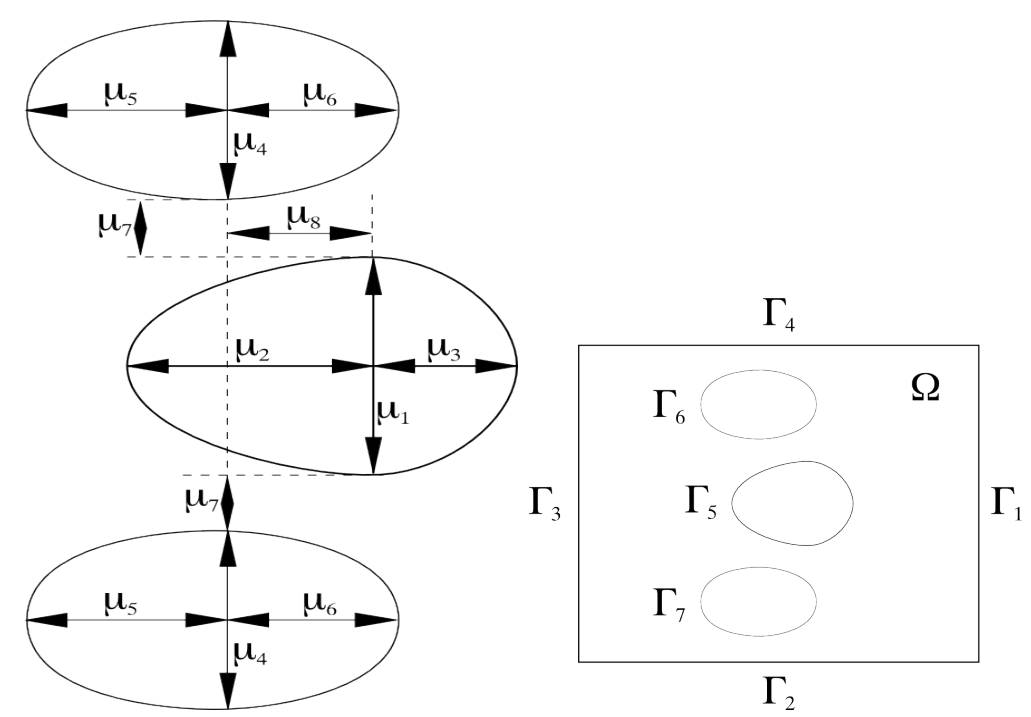

Fig. 5. Parametrized geometrical configuration with three bodies (left) and schematic domain (right).
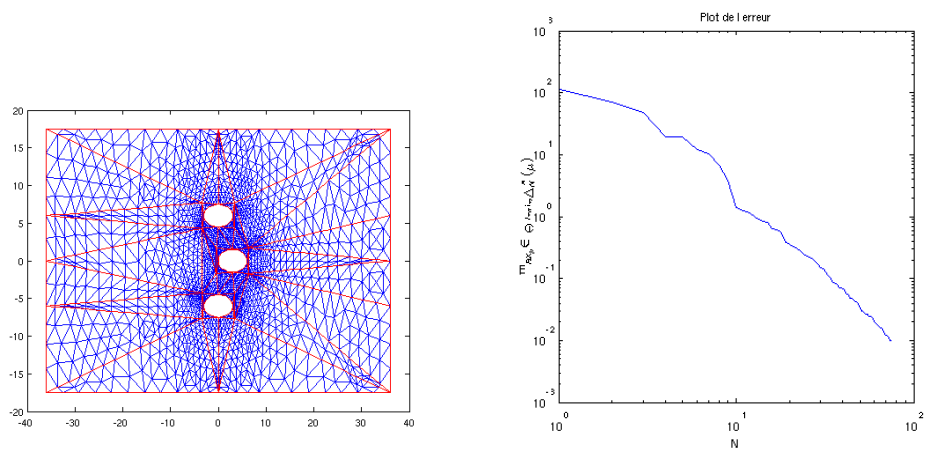

Fig. 6. Mesh and triangulation of the considered configuration (left); convergence of the greedy algorithm over a large sample of parameters: maximum $\Delta_{N}^{e n}(12)$ as a function of $N$ for the same configuration (right).

We report in Table 2 offline and online computational times for the last test. We can see that the average online times for the solution of the problem for a certain $\mu \in \mathcal{D}$ is less than $0.5 \%$ compared with the offline computational times needed to set the geometry, build the mesh and compute FE solutions (and error bound ingredients). The method is well suited, efficient and reliable for the solution of PDEs in parametrized geometries 
in the many query context.

Table 2. Computational times for the third test.

\begin{tabular}{|c|c|}
\hline phase & computational times $(\mathrm{s})$ \\
\hline offline & 4853.7 \\
online & 16.6 \\
\hline ratio & $0.34 \%$ \\
\hline
\end{tabular}

\section{Conclusion}

We have described the basic elements of the reduced basis method and introduced simple problems dealing with a steady potential flow around parametrized bodies. The offline-online computational decomposition strategy is crucial to achieve computational economies of at least two orders of magnitude in the many query context and in a repetitive computational environment. A posteriori error bounds and greedy algorithm convergence prove the reliability of the methodology.

\section{References}

1. K. Ito and S. Ravindran, Journal of Computational Physics 143, 403 (1998).

2. A. Patera and G. Rozza, Reduced Basis Approximation and A Posteriori Error Estimation for Parametrized Partial Differential Equations, (MIT Pappalardo Graduate Monographs Series in Mechanical Engineering, available at http://augustine.mit.edu, (C)Massachusetts Institute of Technology 200608).

3. J. S. Peterson, SIAM Journal of Scientific and Statistical Computing 4, 777 (1989).

4. T. A. Porsching, SIAM Journal of Numerical Analysis 24, 1277 (1987).

5. C. Prud'homme, D. Rovas, K. Veroy, L. Machiels, Y. Maday, A. Patera and G. Turinici, Journal of Fluids Engineering 124, 70 (2002).

6. G. Rozza, D. Huynh and A. Patera, Archives Computational Methods in Engineering 15, 229 (2008).

7. A. Quarteroni and A. Valli, Numerical Approximation of Partial Differential Equations (Springer-Verlag, 1997).

8. D. Huynh, G. Rozza, S. Sen and A. Patera, Comptes Rendus de l'Académie des Sciences Paris Sér I 345, 473 (2007).

9. W. Rheinboldt, Nonlinear Analysis, Theory, Methods and Applications 21, 849 (1993).

10. R. L. Panton, Incompressible Flow, 3rd edn. (John Wiley \& Sons, Inc., 2005).

11. D. Huynh, N. Nguyen, G. Rozza and A. Patera, rbMIT Software and Documentation. (C) Massachusetts Institute of Technology, (2007-08). Available at http://augustine.mit.edu. 